\title{
Precision Micro Assembly of Optical Components on MID and PCB
}

\author{
Jonathan Seybold, Ulrich Kessler, Karl-Peter Fritz, and Heinz Kück \\ Hahn-Schickard-Gesellschaft, Institute for Micro Assembly Technology (HSG-IMAT), \\ Stuttgart, Germany \\ \{seybold, kessler, fritz, kueck\} @hsg-imat.de
}

\begin{abstract}
At HSG-IMAT the precision assembly of micro systems became increasingly important in recent years. The challenge is to reach a high accuracy of about $10 \mu \mathrm{m}$ between the assembled elements. At the same time it is important that the applied processes are suitable for low cost manufacturing. Attempts were made to achieve these requirements by means of automatic assembly processes based on MID and PCB. The results of the development at HSGIMAT are shown in this contribution using the example of an optical module of a rotary encoder. Thereby, the assembly of the laser diode and lens requires special attention to achieve the specified positioning tolerances.
\end{abstract}

Keywords: Optical module, laser diode, VCSEL, sensor, rotary encoder, precision assembly, low cost, miniaturization, MID.

\section{Indroduction}

Micro assembly of optical elements often requires high precision joining operations during the assembly of the components. In this contribution the assembly of the optical components of a module for rotary encoders is described. Rotary encoders are used in many industrial applications for positioning and motion control. Thereby, optical encoders are usually used for high precision measurements [1].

In this case, an optical encoder was designed and built up with an alternative functional principle, which was newly developed at HSG-IMAT. The first feature is the precisely micro structured plastic disc as solid measure similar to a compact disc (CD) or digital versatile disc (DVD). The disc of the encoder is made of polycarbonate and is manufactured by injection moulding at low manufacturing costs.

The second feature is that the encoder contains an optical module with a laser diode, a lens and photo diodes. The assembly of this optical module is described in this paper. The motivation of the research is to reach a plug and play assembly of the encoder itself which leads to a low cost encoder but in spite of this, the encoder has also a high resolution [2]. To achieve this objective, a relative positioning accuracy of \pm 10 $\mu \mathrm{m}$ between laser diode and lens is required in the optical module. The reason for this is, that all critical tolerances than are included in the optical module itself.

To achieve the required accuracy of $\pm 10 \mu \mathrm{m}$ between laser diode and lens two different approaches were proposed. On the one hand an encoder with a MID optical 
module was designed and built up [3]. In this case, the challenge is to reach the required accuracy during the assembly process of the laser diode. On the other hand the optical components were directly assembled on PCB with automated assembly processes [4]. In this case, the challenge is to reach the required accuracy during the assembly process of the lens.

\section{Optical Module on MID}

The first optical module was based on Moulded-Interconnect-Device (MID) technology using the LPKF LDS® process [5]. The optical module contains one laser diode (VCSEL), one lens and five photo diodes. First, the laser diode was manually attached exactly to the center of the MID-header within the required tolerance of $\pm 10 \mu \mathrm{m}$ using a Finetech Fineplacer. Accordingly, the five photodiodes were manually attached whereby the cathode was connected with conductive adhesive. All dies were wirebonded directly to the MID-substrate to connect the anode. Afterwards, the lens was assembled manually with form closure on the MID-header. In the final step, the optical module was connected by conductive adhesive to a PCB on which the electrical processing of the signals was performed. Fig. 1 shows on the left side the assembled and wire-bonded dies on the MID-substrate and below the assembled lens. The right side shows the completely assembled optical module together with the PCB. The photo diodes with an edge length of $1.4 \mathrm{~mm}$ can be used as scale.

The contour of the lens has been designed to fit on the MID-header at the bottom and to carry an aperture at the top. The lens was made of PMMA and was manufactured by ultra-precise diamond turning. For larger quantities the lens can also be manufactured by injection moulding.
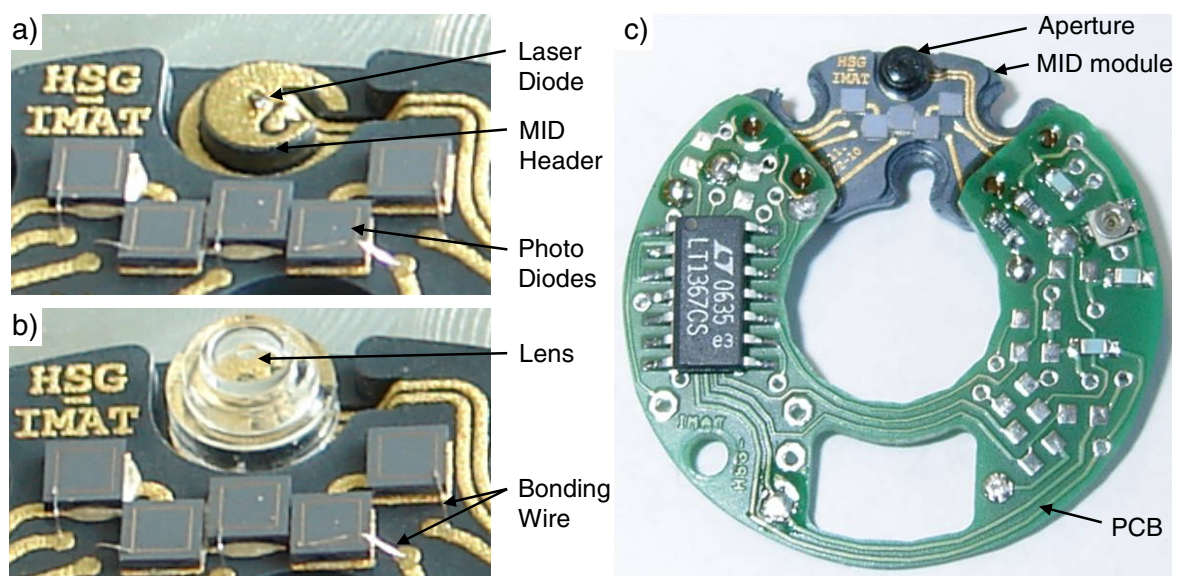

Fig. 1. a) MID-substrate with assembled laser diode and photo diodes; $b$ ) MID-substrate with assembled lens; c) MID module inclusive the assembled aperture connected to the PCB 


\section{Optical Module on PCB}

\subsection{Assembly of the Laser Diode}

Since the first results of the output signals of this encoder were very promising, further investigations on this encoder technology including the precise assembly technology were started at HSG-IMAT. The next step was to apply the optical elements on PCB. Therefore the assembly of the laser diode has been studied in detail. Because of its small size of only $200 \times 200 \times 150 \mu \mathrm{m}^{3}$ the automated assembly of the laser diode is a challenge. The investigations were conducted on the automated micro assembly system Vico Base from Häcker Automation GmbH.

At first, different vacuum pick up tools for the assembly of the laser diode were tested. The best results were achieved with a pick-up tool with conical shape which holds the laser diode at its edges. Thereby the laser diode is held in a defined position in the tool through a self centering effect because of the conical shape of the tool [6]. This allows to assemble the laser diode without a bottom side referencing step. It has become evident, that a bottom side referencing of the laser diode leads to a loss of positioning accuracy due to the poor quality of the bottom side edges in combination with an insufficient resolution of the inspection system. Fig. 2 shows on the left side the laser diodes on blue tape in the state as delivered. The right side schematically shows the pick-up process of the laser diodes from the blue tape.

The assembly process of the laser diode runs fully automatic in several steps. First the reference marks on the PCB are detected with the inspection camera. Subsequently the conductive adhesive is applied to the target position by a stamping process.

Afterwards the respective pick-up position of the laser diode on blue tape is detected exactly with the inspection camera and the laser diode is picked up and held in the pick-up tool by vacuum. After moving to the target position and the assembly the vacuum is switched off and the pick-up tool moves to the starting position. Finally, the thermal curing process of the adhesive is carried out in an oven.

The other studied tools were either made from rubber or metal and had flat surfaces, so that the tool touches the top side of the chip. With the metal tool the surface of the laser diode was damaged and with the rubber tool no stable pick-up and positioning process was possible due to the small rubber tip, which proved to be too instable.

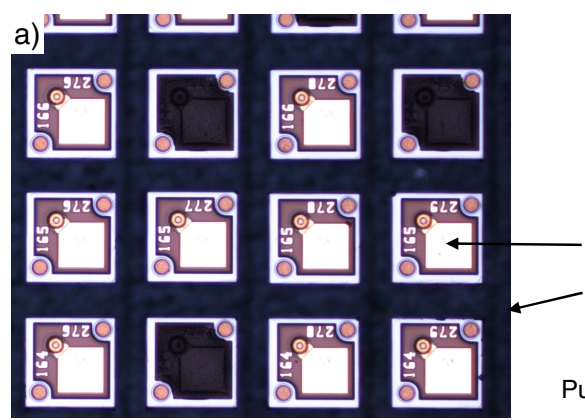

b)

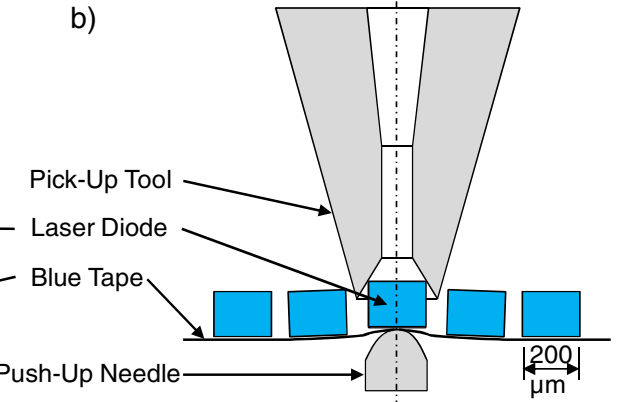

Fig. 2. a) Laser diodes on blue tape; $b$ ) Schematic pick-up process from blue tape 


\subsection{Positioning Accuracy of the Laser Diode}

To investigate and optimize the positioning accuracy several $3 \times 3$ laser diode arrays were assembled on an experimental PCB using different setups. In detail the shape and size of the reference marks on the PCB were varied and also the procedure of the camera inspection of the laser diode before the pick-up process from blue tape. Fig. 3 shows such an assembled laser diode array.

It could be shown, that the automatic inspection works best with rectangular or linear contrast transitions. Therefore rectangular reference marks should be preferred over circular or crosswise marks. Accordingly the inspection of the laser diode works better when the rectangular structures of the surface are used instead of the circular alignment marks. Furthermore it has been shown that for highly precise assembly it can be advantageous to use the reference marks on the PCB only for assembly of the first die if there is more than one chip to be assembled. For the subsequent assembly steps it is then possible to use the first assembled die as reference mark. In this way, the more precise lithographic structures of the die instead of the etched reference marks on the PCB, which are less precise, are used to achieve the required accuracy.

The $3 \times 3$ laser diode array in Fig. 3 was assembled according to this principle. The first laser diode was assembled in relation to the reference mark on PCB. The other laser diodes were assembled in relation to the first laser diode, whereby for each laser diode a new inspection step of the reference laser diode was done. In order to investigate the quality of the optical referencing, the PCB was loaded to and unloaded from the machine between the assembly of each laser diode.

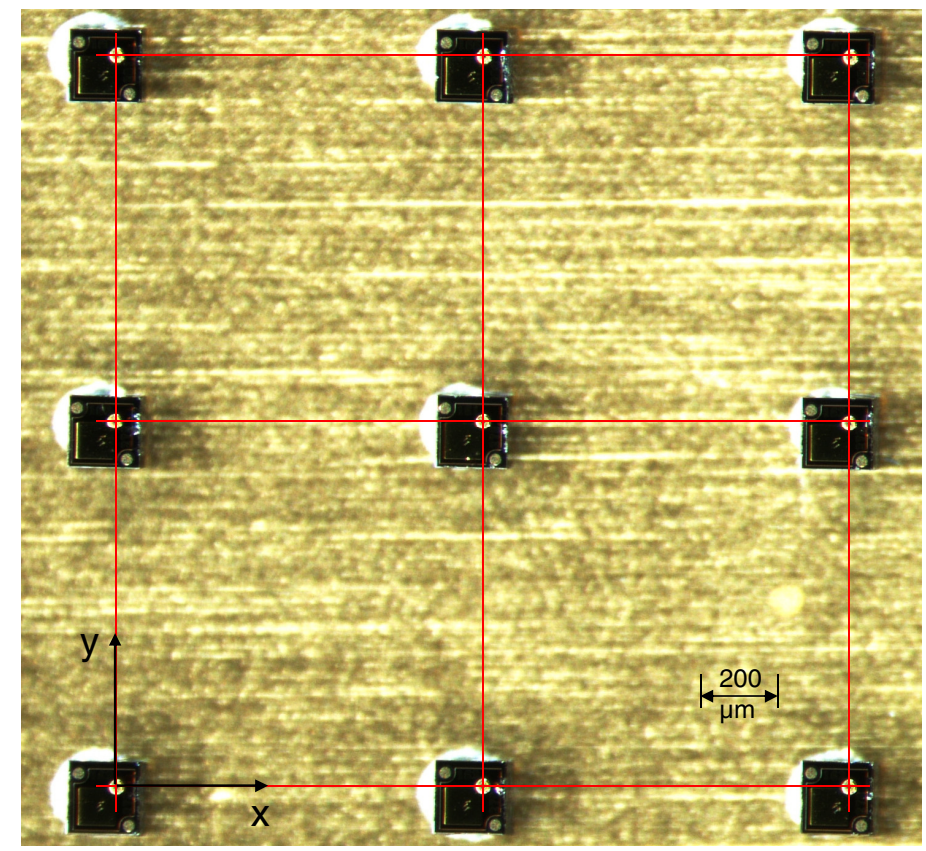

Fig. 3. 3x3 Laser diode array on PCB 
The positions of the facets of the laser diodes were measured before and after the curing process on a coordinate measuring machine und compared with the intended target positions. The diagram in Fig. 4 shows the measured displacement of the facets of the laser diodes from their target positions. After curing, the maximum displacement was found to be $\pm 7 \mu \mathrm{m}$, the standard deviation was $<3.5 \mu \mathrm{m}$. Altogether five $3 \times 3$ laser diode arrays were assembled.

The die attach of the photo diodes is easier due to their larger size. In addition, the sensor concept doesn't require the same level of accuracy as it is with the laser diode and lens. The assembly could therefore be done using a rubber tool.

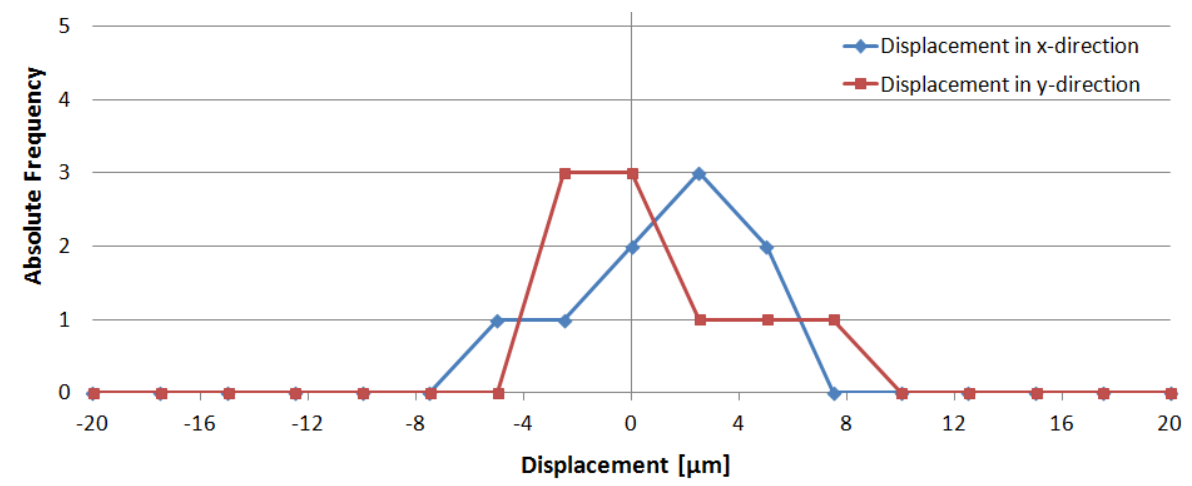

Fig. 4. Frequency distribution of the displacement in $\mathrm{x}$ - and $\mathrm{y}$-direction

\subsection{Assembly of the Lens}

The assembly of the lens has similar requirements concerning the accuracy like the laser diode. First, UV curable adhesive is transferred by a ring-shaped stamp to the PCB. Then the lens is picked up with a conical pick-up tool which assures the defined alignment of the lens in the pick-up tool. The target position of the lens on the PCB equates to the facet of the laser diode. Therefore an inspection step of the laser diode is done. Then the lens is assembled and the adhesive is cured through UV radiation. To ensure the correct distance between laser diode and lens, on the bottom side of the lens a gap for the adhesive is defined. Fig. 5 shows schematically the assembly process of the lens on the PCB.

The challenge in this case is to find the center of the lens before the pick-up step. Because of the clear optical surfaces, it is difficult for the inspection camera to find sufficient contrast at the edges. For this case, a ring at the top side of the lens was designed. When manufacturing the lens by injection moulding, the ring shaped plane can be arranged with a mat surface to enhance the contrast.

The assembled optical module on PCB is shown in Fig. 6. Visible are the PCB with the electronic components, which were assembled first, the photo diodes and the lens. The laser diode is located below the lens. The photo diodes with an edge length of $1.4 \mathrm{~mm}$ can be used as scale.

The misalignment between laser diode and the assembled lens is determined as the difference between the center of the lens, measured by optical inspection and the centre 


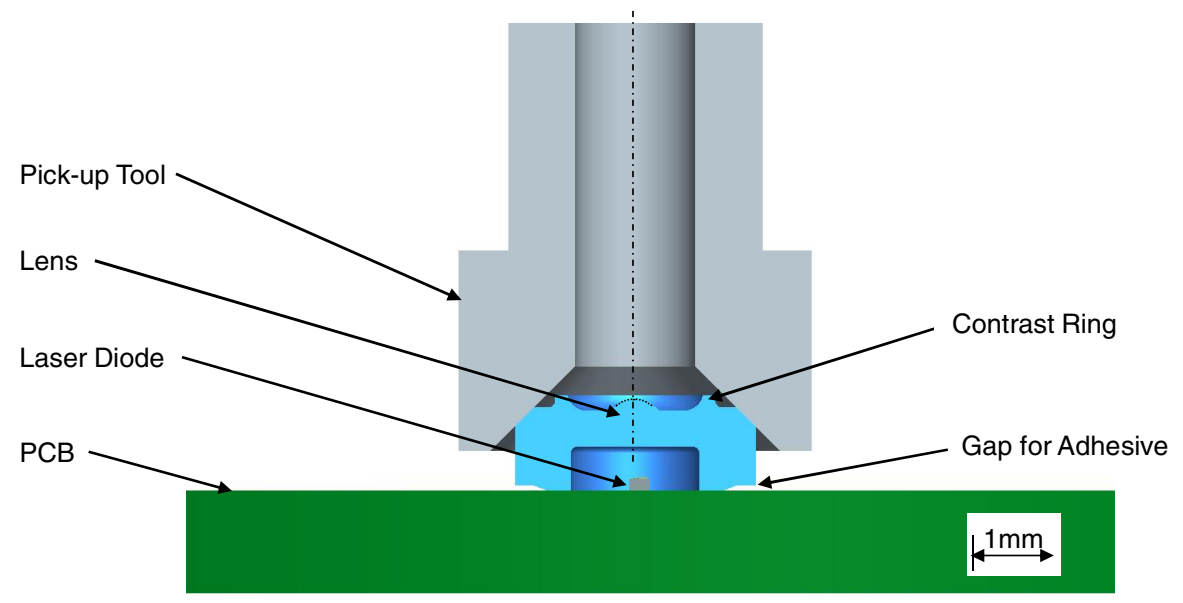

Fig. 5. Schematic assembly process of the lens on PCB

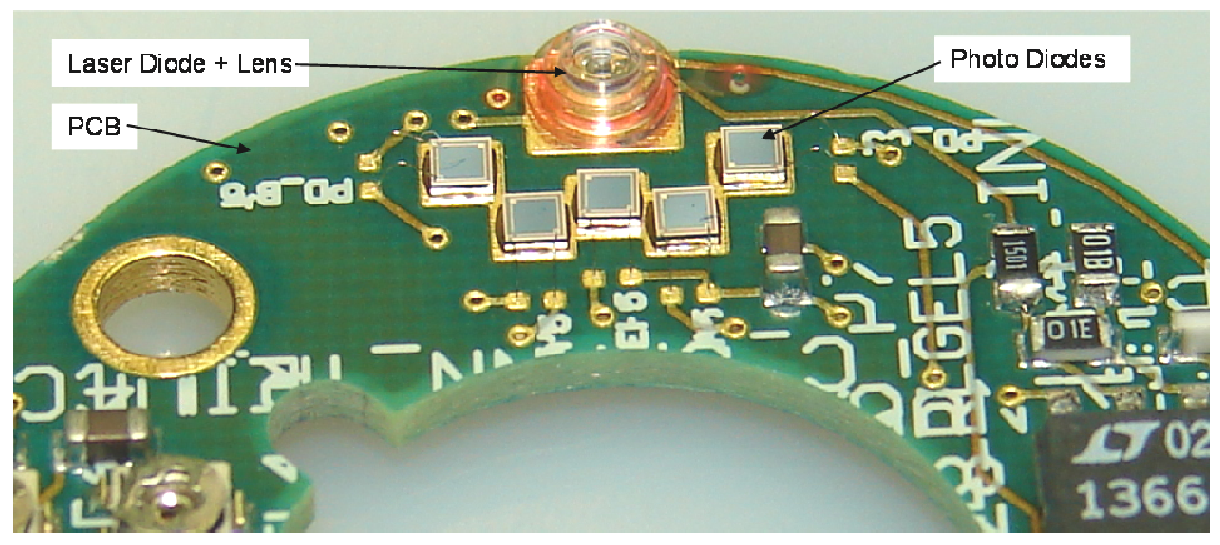

Fig. 6. Complete assembled optic module on PCB
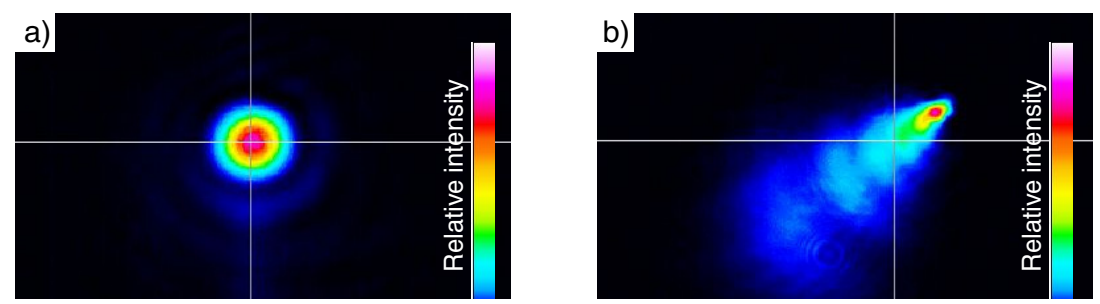

Fig. 7. Beam profile of a correct assembled lens $(a)$ and of a misaligned lens $(b)$

of the facet of the laser diode which can be seen as image through the lens in its focal plane. Another method is to consider the beam profile of the spot. Fig. 7 shows on the left side the beam profile with a correctly aligned lens and on the right side a misaligned lens. Shown is the relative intensity of the laser beam in its beams waist plane in 
which is the encoder disc with its solid measure too. A misaligned lens causes of course poor signal quality or even total failure of the rotary encoder when the misalignment is too large.

\section{Conclusion and Outlook}

A miniature optical module for a rotary encoder was designed and built up first based on MID and further on PCB. The optical module includes one laser diode, one lens and several photo diodes. The positioning accuracy of the assembled optical components was analysed. The result is that assembly within $\pm 10 \mu \mathrm{m}$ is possible under the described circumstances. Further investigations should be done under the condition of large scale manufacturing to confirm this result.

HSG-IMAT will continue to work in the field of precision assembly. For example automated active alignment of the lens by beam control and a higher resolution of the inspection camera of the 3-D assembly machine are two issues, which will bring further progress in precision assembly.

In addition, it is planned at HSG-IMAT to investigate and develop forward the 3-D assembly on MID. This enables to use the advantage of the MID-technology in reference to the degree of freedom in 3-D. From this point of view, this technology allows to design mechanical joining elements which can be used for the purpose of alignment or also actuators and sensors with functions based on 3-D, for example 3-D magnetic field sensors.

\section{References}

1. Schaeffler, K.G.: Magnetisch versus optisch; Winkel-Mess-Systeme für Rundtischachsen im Vergleich (2008), http: / / www. schaeffler. com

2. Hopp, D.: Inkrementale und absolute Kodierung von Positionssignalen diffraktiver optischer Drehgeber; Dissertation Universität Stuttgart (2012)

3. AiF-Vorhaben Nr. 219 ZN: Untersuchungen zu einem hochauflösenden optischen Drehwinkelsensor in Low-Cost-Bauweise; Institut für Mikroaufbautechnik; Hahn-SchickardGesellschaft (2008)

4. AiF-Vorhaben Nr. $349 \mathrm{ZN}$ : Untersuchungen zu kostengünstigen absolut und inkremental kodierten optischen Drehgebern mit justagefreier Endmontage und mikrostrukturierter diffraktiver Maßverkörperung aus Kunststoff; Institut für Mikroaufbautechnik; HahnSchickard-Gesellschaft (2012)

5. Forschungsvereinigung 3-D MID e.V.: 3D-MID Technologie. München, Carl Hanser Verlag (2004)

6. Die Attachment and Fluid Dispensing; Brochure; SPT Roth Ltd. (2013),

http: //www. smallprecisiontools.com 